\title{
A Comparison of the Imaging Characteristics and Microregional Distribution of 4 Hypoxia PET Tracers
}

\author{
Sean Carlin ${ }^{1}$, Hanwen Zhang ${ }^{1}$, Megan Reese ${ }^{1}$, Nicholas N. Ramos ${ }^{1}$, Qing Chen ${ }^{2}$, and Sally-Ann Ricketts ${ }^{3}$ \\ ${ }^{1}$ Radiochemistry and Imaging Sciences Service, Department of Radiology, Memorial Sloan-Kettering Cancer Center, New York, New \\ York; ${ }^{2}$ Department of Medical Physics, Memorial Sloan-Kettering Cancer Center, New York, New York; and ${ }^{3}$ AstraZeneca, \\ Personalised Healthcare and Biomarkers, Macclesfield, Cheshire, United Kingdom
}

\begin{abstract}
We compared the imaging characteristics and hypoxia selectivity of 4 hypoxia PET radiotracers $\left({ }^{18} \mathrm{~F}\right.$-fluoromisonidazole $\left[{ }^{18} \mathrm{~F}-\mathrm{FMISO}\right],{ }^{18} \mathrm{~F}-$ flortanidazole [ $\left.{ }^{18} \mathrm{~F}-\mathrm{HX} 4\right]$ ], ${ }^{18} \mathrm{~F}$-fluoroazomycin arabinoside $\left[{ }^{18} \mathrm{~F}-\mathrm{FAZA}\right]$, and ${ }^{64} \mathrm{Cu}$-diacetyl-bis(N4-methylsemicarbazone) $\left[{ }^{64} \mathrm{Cu}-\mathrm{ATSM}\right]$ ) in a single murine xenograft tumor model condition using small-animal PET imaging and combined ex vivo autoradiography and fluorescence immunohistochemistry. Methods: Nude mice bearing SQ20b xenograft tumors were administered 1 of 4 hypoxia PET tracers and images acquired $80-90 \mathrm{~min}$ after injection. Frozen sections from excised tumors were then evaluated for tracer distribution using digital autoradiography and compared with histologic markers of tumor hypoxia (pimonidazole, carbonic anydrase 9 [CA9]) and vascular perfusion (Hoechst 33342). Results: The highest tumor uptake was observed with ${ }^{64} \mathrm{Cu}$-ATSM (maximum standardized uptake values [SUVmax], $1.26 \pm 0.13$ ) and the lowest with ${ }^{18} \mathrm{~F}-\mathrm{FAZA}$ (SUVmax, $0.41 \pm 0.24$ ). ${ }^{18} \mathrm{~F}-\mathrm{FMISO}$ and ${ }^{18} \mathrm{~F}-\mathrm{HX} 4$ had similar intermediate tumor uptake (SUVmax, $0.76 \pm 0.38$ and $0.65 \pm 0.19$, respectively). Digital autoradiographs of hypoxia tracer distribution were compared pixel by pixel with images of immunohistochemistry stains. The fluorinated nitroimidazoles all showed radiotracer uptake increasing with pimonidazole and CA9 staining. ${ }^{64} \mathrm{Cu}$-ATSM showed the opposite pattern, with highest radiotracer uptake observed in regions with the lowest pimonidazole and CA9 staining. Conclusion: The fluorinated nitroimidazoles showed similar tumor distributions when compared with immunohistochemistry markers of hypoxia. Variations in tumor standardized uptake value and normal tissue distribution may determine the most appropriate clinical setting for each tracer. ${ }^{64} \mathrm{Cu}$-ATSM showed the highest tumor accumulation and little renal clearance. However, the lack of correlation between ${ }^{64} \mathrm{Cu}$-ATSM distribution and immunohistochemistry hypoxia markers casts some doubt on the hypoxia selectivity of ${ }^{64} \mathrm{Cu}$-ATSM.
\end{abstract}

Key Words: autoradiography; animal imaging; PET; hypoxia; immunofluorescence

J Nucl Med 2014; 55:515-521

DOI: $10.2967 /$ jnumed.113.126615

\section{$\mathbf{T}$} he presence of regions of low tumor oxygen partial pressure $\left(\mathrm{pO}_{2}\right)$ have long been associated with resistance to radio- and chemotherapy and increased incidence of metastasis and rates of disease

Received May 22, 2013; revision accepted Oct. 25, 2013.

For correspondence or reprints contact: Sean Carlin, Radiochemistry and Imaging Sciences Service, Memorial Sloan-Kettering Cancer Center, 415 E. 68th St., New York, NY 10065.

E-mail: carlins@mskcc.org

Published online Feb. 3, 2014

COPYRIGHT (C 2014 by the Society of Nuclear Medicine and Molecular Imaging, Inc. recurrence. This phenomenon was attributed to a direct effect of $\mathrm{pO}_{2}$ on the efficacy of external-beam radiation (termed the oxygen enhancement effect) (1). Recent studies have also highlighted the role of the cellular oxygen-sensing mechanisms, in particular the hypoxiainducible factor (HIF) transcription factors, in governing tumor phenotype and response to therapy (2). Although the influence of tumor hypoxia on disease progression and treatment response is becoming increasing clear, there is no widespread clinical utility for the determination of $\mathrm{pO}_{2}$ in solid tumors. This is in part due to the methods currently available to measure $\mathrm{pO}_{2}$, none of which are completely appropriate as universal biomarkers of tumor hypoxia. Polarographic electrodes inserted directly into tumors can provide absolute $\mathrm{pO}_{2}$ measurements but can only practically be performed on easily accessible tumor sites. The systemic administration of 2-nitroimidazole-based hypoxia tracers such as pimonidazole and EF5, followed by immunohistochemical detection, has also been previously used (3). These compounds are reduced and specifically retained in hypoxic tumor cells, allowing detection and quantitation of hypoxic regions at the microscopic level. However, in common with polarographic electrode measurements, the method is invasive and subject to potential sampling errors. The employment of endogenous histologic biomarkers of tumor hypoxia has also been widely examined, with the markers carbonic anhydrase $9(\mathrm{Ca} 9)$ and lysyl oxidase showing the most promise (4). Employment of such markers can be complicated by cell-type-specific expression and nonuniform response to changes in the underlying $\mathrm{pO}_{2}$, nonhypoxic regulation of expression, and a temporal mismatch between changes in $\mathrm{pO}_{2}$ and the corresponding change in marker expression (5).

When compared with these methods, PET imaging using hypoxia-selective tracers is an attractive option for tumor $\mathrm{pO}_{2}$ assessment. Several hypoxia PET tracers have been developed, based mainly on radiochemical derivatives of the 2-nitroimidazole and (bis)thiosemicarbazone structures (6). PET imaging is generally conducted soon after tracer administration (reducing temporal mismatch) and allows quantitative imaging of the whole tumor (reducing sampling error). Although cellular uptake of these tracers is passive, an enzyme-mediated reduction step is crucial to their hypoxia selectivity. However, in the case of 2-nitroimidazole compounds, bioreductive enzyme expression is rarely rate-limiting, and $\mathrm{pO}_{2}$ is the primary determinant of tracer retention (7). Significant efforts have been made to optimize hypoxia PET image contrast, primarily by modifications to the chemical structures of the radiotracers resulting in altered pharmacokinetics (8). In general, the more lipophilic compounds display rapid tissue equilibration and higher first-pass tumor uptake rates but slower background clearance and higher liver accumulation. The hydrophilic compounds display the reverse characteristics: rapid renal clearance, lower liver uptake, 
and reduced production of metabolites, at the cost of lower absolute uptake and more heterogeneous tumor distribution.

There are few published studies that directly compare the performance of the PET hypoxia tracers relative to each other under controlled conditions. Those that have been conducted have used sequential tracer administration to the same patient or animal, separated by periods of up to $3 \mathrm{~d}$. However, acute changes in tumor hypoxia can potentially introduce uncertainly into studies using sequential tracer administration. The aim of this study was to compare the imaging characteristics and hypoxia specificity of 4 hypoxia PET tracers under standardized conditions in the same animal xenograft model.

\section{MATERIALS AND METHODS}

\section{Animal Models and Cell Culture}

SQ20b, HT29, and HCT116 cells were cultured in Dulbecco modified Eagle medium supplemented with $10 \%$ (v/v) fetal bovine serum, penicillin $(100 \mathrm{U} / \mathrm{mL})$, and streptomycin $(100 \mu \mathrm{g} / \mathrm{mL})$ and were maintained at $37^{\circ} \mathrm{C}$ in a humidified atmosphere containing $5 \% \mathrm{CO}_{2}$.

Animal studies were conducted in compliance with protocols approved by the Memorial Sloan-Kettering Cancer Center (MSKCC) Institutional Animal Care and Use Committee. In vivo experiments were performed using 6- to 8-wk-old female athymic NCr-nu/nu mice. Tumors were initiated by injecting $5 \times 10^{6}$ tumor cells in $0.1 \mathrm{~mL}$ of phosphate-buffered saline subcutaneously into the hind limb. Radiotracer experiments were performed when tumors reached approximately $1 \mathrm{~cm}$ in average diameter, which occurred typically 3-4 wk after initiation.

\section{Radiotracers and Histologic Markers}

For ${ }^{64} \mathrm{Cu}$-diacetyl-bis (N4-methylsemicarbazone) $\left({ }^{64} \mathrm{Cu}\right.$-ATSM) highspecific-activity ${ }^{64} \mathrm{Cu}$ radioisotope was obtained from Washington University School of Medicine, and ligand synthesis and labeling was conducted as previously described (9). A radiochemical purity of $99 \%$ was assessed by instant thin-layer chromatography. ${ }^{18} \mathrm{~F}-$ fluoride was produced on the MSKCC cyclotron (TR19/9; EBCO Technologies, Inc.). ${ }^{18} \mathrm{~F}$-fluoromisonidazole $\left({ }^{18} \mathrm{~F}\right.$-FMISO) (specific activity, $370 \mathrm{MBq} / \mu \mathrm{g}[10 \mathrm{mCi} / \mu \mathrm{g}])$ was prepared according to the method of Grierson et al. (10) by the Cyclotron and Radiochemistry Service at MSKCC. ${ }^{18} \mathrm{~F}$-fluoroazomycin arabinoside $\left({ }^{18} \mathrm{~F}-\mathrm{FAZA}\right)$ was prepared as described according to the method of Maier et al. (11). The radiochemical purity of ${ }^{18} \mathrm{~F}$-FMISO and ${ }^{18} \mathrm{~F}$-FAZA, determined by highperformance liquid chromatography, was greater than $98 \% .{ }^{18} \mathrm{~F}$-flortanidazole $\left({ }^{18} \mathrm{~F}-\mathrm{HX} 4\right)$ was obtained from Siemens PETNET solutions (Siemens Medical Solutions, USA) and radiochemical purity greater than $98 \%$ by high-performance liquid chromatography.

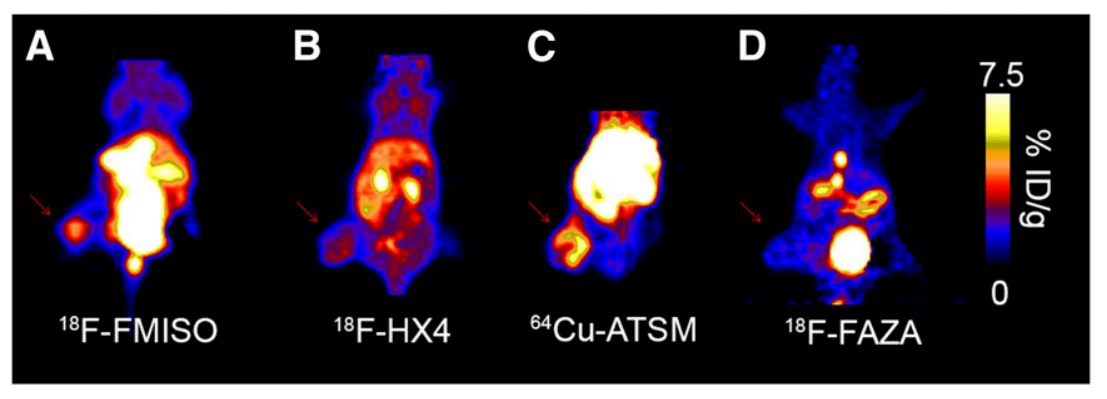

FIGURE 1. Representative coronal images obtained from tumor-bearing mice administered each of the hypoxia PET tracers indicated. Images were normalized to same scale and window selected to allow comparison of relative tumor uptake. Red arrow indicates location of tumor on hind limb. $\% \mathrm{ID} / \mathrm{g}=$ percentage injected dose per gram.
Animals were intravenously coadministered 9.25-33.3 MBq (250$900 \mu \mathrm{Ci}$ ) of the respective tracer along with pimonidazole hydrochloride (80 mg/kg; Hypoxyprobe-1 [Natural Pharmacia International Inc.]), in a final injection volume of $200 \mu \mathrm{L}$. Hoechst 33342 trihydrochloride (Sigma; $1 \mathrm{mg}$ in $100 \mu \mathrm{L}$ of physiologic saline) was injected $1 \mathrm{~min}$ before sacrifice.

\section{PET Imaging}

Mice were anesthetized using a $1 \%$ isoflurane-to-air mixture and imaged prone with either an R4 or a Focus 120 dedicated small-animal PET scanner (Concorde Microsystems Inc.), an energy window of 350$700 \mathrm{keV}$, and a coincidence timing window of $6 \mathrm{~ns}$. Images were acquired over a period of $10 \mathrm{~min}$, between 80 and $90 \mathrm{~min}$ after administration. Images were reconstructed using the ordered-subset expectation maximization 2-dimensional algorithm. Attenuation and scatter correction were not applied. Mean and maximum standardized uptake values (SUVs) were derived as previously described (12), using the formula [\% $\mathrm{ID} / \mathrm{g} \times$ body mass $(\mathrm{g})] / 100(\% \mathrm{ID} / \mathrm{g}$ is percentage injected dose per gram).

\section{Autoradiography and Fluorescence Microscopy}

After imaging, a 28-gauge angiocatheter was placed in the tumor to assist with subsequent image registration. Animals were then sacrificed, tumors were excised and embedded in optimal-cutting-temperature mounting medium (Sakura Finetek) and frozen on dry ice, and a series of $10-\mu \mathrm{m}$ frozen sections was cut. To determine radiotracer distribution, digital autoradiography was performed by placing tissue sections in a film cassette against a phosphor imaging plate (Fujifilm BASMS2325; Fuji Photo Film) for an appropriate exposure period at $-20^{\circ} \mathrm{C}$. Phosphor imaging plates were read at a pixel resolution of $50 \mu \mathrm{m}$ with a BAS-1800II Bio-Imaging Analyzer (Fujifilm Medical Systems). After autoradiographic exposure, the same frozen sections were then used for fluorescence staining and microscopy.

Immunofluorescence staining for pimonidazole and carbonic anydrase 9 (CA9) was performed essentially as previously described (3), the major difference being the use of a rabbit polyclonal antipimonidazole primary antibody (Natural Pharmacia International Inc.). Secondary detection was performed using goat antirabbit Alexa-488 (for pimonidazole, 1:100) and goat antihuman Alexa-568 (for CA9, 1:100) (Life Technologies Inc., USA) in blocking buffer. Images were acquired at $\times 40$ magnification using a BX60 fluorescence microscope (Olympus America, Inc.) equipped with a motorized stage (Prior Scientific Instruments Ltd.) and CC12 camera (Olympus). Whole-tumor montage images were obtained by acquiring multiple fields at $\times 40$ magnification, followed by alignment using MicroSuite Biologic Suite (version 2.7; Olympus).

\section{Image Registration}

Fluorescence and autoradiographic data were registered using Adobe Photoshop (version CS3). The microscope objective calibration factor was used to normalize all images to the same pixel resolution, allowing upsampling of the autoradiographic image or downsampling of the fluorescence images as required. A combination of visual landmarks (tumor edges) and holes resulting from angiocatheter placement were used to register the image datasets. Because the same sections were used for both autoradiography and fluorescence imaging, subsequent registration of each image set was free from registration errors.

\section{Pixel Rebinning and \\ Scatterplot Generation}

Pixel rebinning was performed using an adaptation of the methods described by Carlin et al. $(13,14)$. Briefly, registered image sets were 
resampled to a pixel size of $50 \mu \mathrm{m}$, corresponding to the native pixel resolution of the digital autoradiography image. Each image was then converted to an 8-bit gray scale, and pixel values with corresponding position were recorded. The data from the digital autoradiography image were designated as independent and the fluorescence image data as dependent. Data were then sorted in ascending order of the independent variable while maintaining the association between independent and dependent values. The dataset was then split into deciles, each containing the same number of data points, that is, the $10 \%$ of the data points lowest in terms of the independent variable, then the next lowest $10 \%$, and so on. The mean and SD of the mean were then calculated for each decile for both independent and dependent variables.

\section{RESULTS}

\section{PET Imaging}

Figure 1 shows representative coronal sections from small-animal PET images of mice bearing hind limb SQ20b subcutaneous xenografts. The PET images were acquired 80-90 min after injection of the indicated radiotracer. ${ }^{18} \mathrm{~F}$-FMISO (Fig. 1A) and ${ }^{18} \mathrm{~F}-\mathrm{HX} 4$ (Fig. 1B) uptake was broadly similar (mean SUV [SUVmean], $0.44 \pm$ $0.17[n=3]$ and $0.42 \pm 0.18[n=5]$, respectively). ${ }^{64} \mathrm{Cu}$-ATSM had a greater tumor uptake than either of the other fluorinated nitroimidazoles (SUVmean, $-0.62 \pm 0.09, n=4$ ). Tumor uptake of ${ }^{18} \mathrm{~F}$-FAZA was lowest of the tracers studied (SUVmean, $-0.27 \pm$ $0.13, n=4) .{ }^{18} \mathrm{~F}$-FMISO showed a marked abdominal distribution, with both the renal and the hepatobiliary clearance routes involved. ${ }^{18} \mathrm{~F}-\mathrm{HX} 4$ distribution appeared to be broadly similar to ${ }^{18} \mathrm{~F}-\mathrm{FMISO}$ but with a more prominent renal uptake and less liver accumulation at this time point, compared with ${ }^{18} \mathrm{~F}$-FMISO. ${ }^{18}$ F-FAZA clearance appeared to be primarily renal, with prominent kidney and bladder activity (Fig. 1C). Low liver and gut activity was observed when compared with ${ }^{18} \mathrm{~F}$-FMISO or ${ }^{18} \mathrm{~F}-\mathrm{HX} 4$. Tumor accumulation of ${ }^{18} \mathrm{~F}-\mathrm{FAZA}$ was lower than of ${ }^{18} \mathrm{~F}-\mathrm{HX} 4$ and ${ }^{18} \mathrm{~F}-\mathrm{FMISO}$, but tumor-background contrast was sufficient to allow clear tumor visualization (Supplemental Fig. 1; supplemental materials are available at http://jnm.snmjournals.org).

${ }^{64} \mathrm{Cu}$-ATSM showed some differences in distribution pattern from the fluorinated nitroimidazoles, with more marked liver accumulation and minimal renal- or bladder-associated activity (Fig. 1D). SUVmean and maximum SUV (SUVmax) at 80-90 min after injection and tumor-to-muscle ratios for each tracer are given in Table 1, with corresponding statistical analysis given in Supplemental Table 1 .

\section{Histology}

Generally, tumor sections were heterogeneous in appearance, with regions of blood vessel-containing stroma visible in the tumor rim becoming less frequent toward the centrally necrotic core (Fig. 2A). Fluorescence microscopy was used to visualize the vascular per-

\section{TABLE 1}

SUVmean, SUVmax, and Tumor-to-Muscle Ratios Derived from Images Obtained at 90 Minutes After Administration

\begin{tabular}{lccl}
\hline Tracer & SUVmean90 & SUVmax90 & Tumor to muscle \\
\hline${ }^{18} \mathrm{~F}-\mathrm{FMISO}$ & $0.445 \pm 0.171$ & $0.758 \pm 0.376$ & $1.61 \pm 0.434(n=3)$ \\
${ }^{18} \mathrm{~F}-\mathrm{HX} 4$ & $0.424 \pm 0.175$ & $0.652 \pm 0.188$ & $1.35 \pm 0.09(n=5)$ \\
${ }^{18} \mathrm{~F}-\mathrm{FAZA}$ & $0.271 \pm 0.132$ & $0.412 \pm 0.236$ & $1.26 \pm 0.342(n=3)$ \\
${ }^{64} \mathrm{Cu}-\mathrm{ATSM}$ & $0.624 \pm 0.089$ & $1.258 \pm 0.133$ & $2.77 \pm 0.463(n=4)$
\end{tabular}

Data are mean \pm SD.

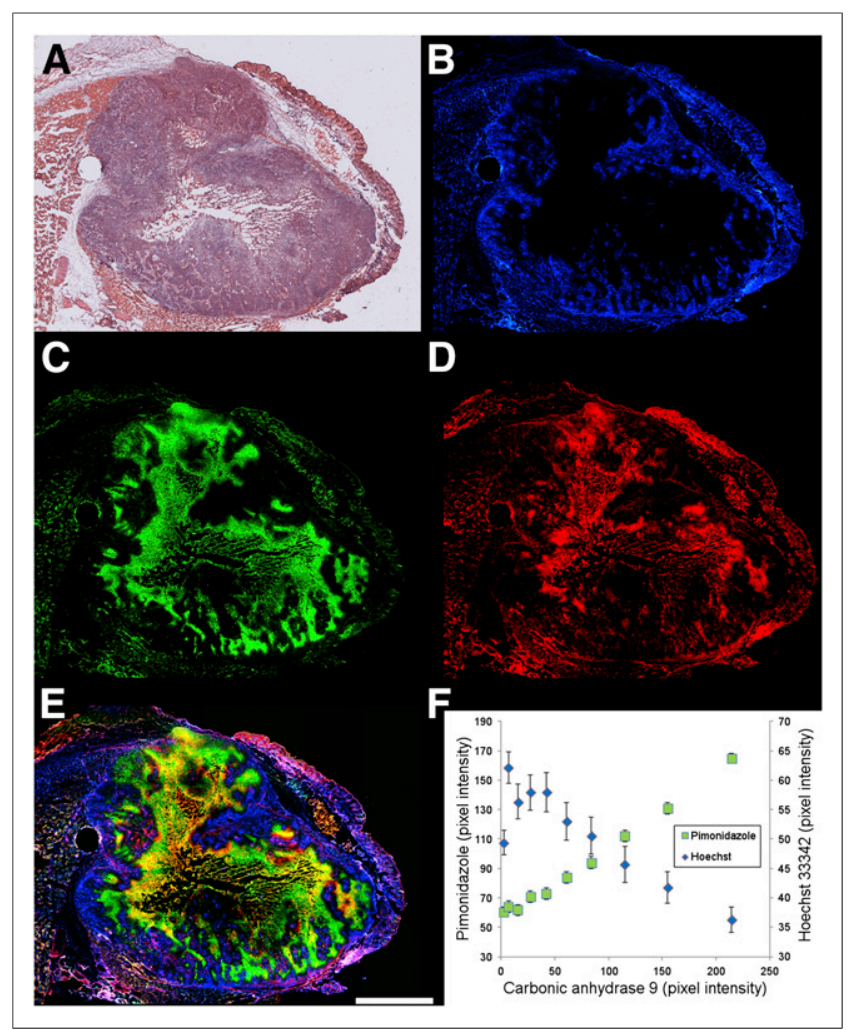

FIGURE 2. Histologic and immunofluorescence staining on SQ20b tumor section. Images of hematoxylin and eosin staining (A), Hoechst 33342 (blue, B), pimonidazole (green, C), and CA9 (red, D) are obtained from same section. Composite registered 3-color image composed of $B-D$ is shown in E. (F) Rebinned scatterplots showing relationship between CA9 pixel intensity and both pimonidazole (green squares) and Hoechst 33342 (blue diamonds). Means and 95\% confidence intervals of mean are shown. Bar in $\mathrm{E}=2 \mathrm{~mm}$.

fusion marker Hoechst 33342 (blue, Fig. 2B), the exogenous hypoxia marker pimonidazole (green, Fig. 2C), and the endogenous marker CA9 (red, Fig. 2D). A single 3-color composite image is shown in Figure 2E. Hoechst 33342 distribution was most prominent in peripheral tumor regions and closely mirrored the appearance of light-staining stroma on the hematoxylin and eosin image. Expression of CA9 was strongest in perinecrotic regions and weakest in regions of high perfusion and stromal density. Pimonidazole distribution was broadly similar to that of CA9 expression, with pronounced perinecrotic staining visible and low uptake in areas positive for Hoechst 33342. Rebinned scatterplots comparing the pixel intensities of each marker showed a positive relationship between CA9-pimonidazole and an inverse relationship between CA9-Hoechst 33342 (Fig. 2F).

\section{Correlative Histology and Autoradiography}

An example of ${ }^{18}$ F-FMISO distribution and corresponding fluorescence markers is shown in Figure 3, where the autoradiograph is shown in 8-bit gray scale (Fig. 3B) from black (lowest activity) to white (highest activity). At both low and high magnifications, there appears a clear correspondence of higher ${ }^{18} \mathrm{~F}$-FMISO uptake with regions of high pimonidazole and CA9 staining. Conversely, regions with prominent Hoechst 33342 staining are associated with lower ${ }^{18} \mathrm{~F}$-FMISO uptake. Similar results were observed for both ${ }^{18}$ F-HX4 and ${ }^{18}$ F-FAZA (shown in Supplemental Fig. 2). In 


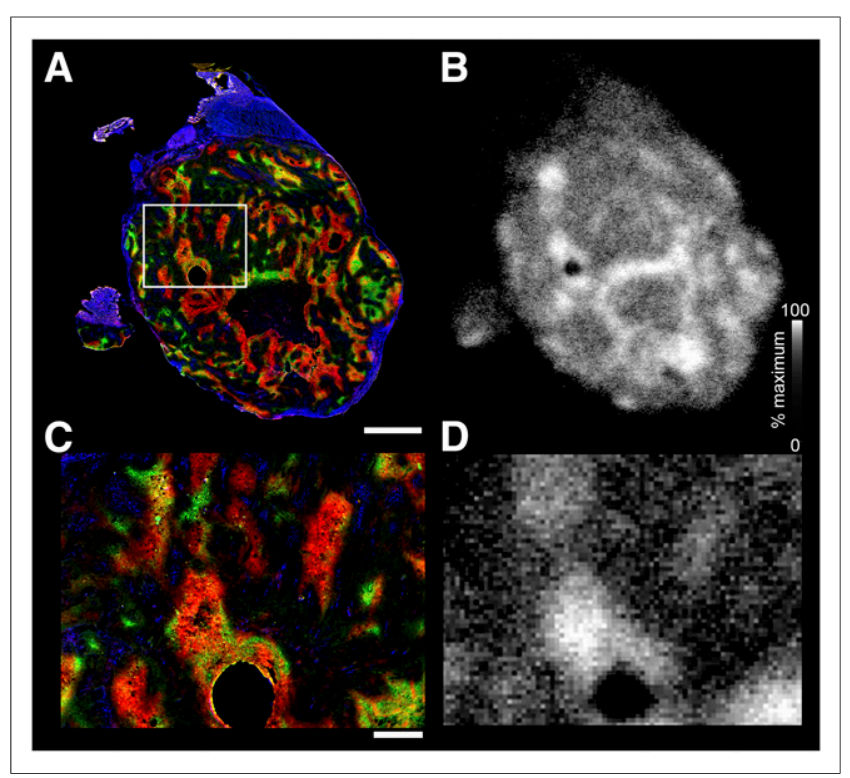

FIGURE 3. Distribution of ${ }^{18} \mathrm{~F}-\mathrm{FMISO}$ versus immunofluorescence makers. (A) Composite registered 3-color image showing Hoechst 33342 (blue), pimonidazole (green), and CA9 (red). Bar in $A=2 \mathrm{~mm}$. (B) Corresponding autoradiographic image of ${ }^{18} \mathrm{~F}$-FMISO distribution from same tumor section. Indicated region in A is shown at high magnification in $C$ and $D$. Circular hole caused by angiocatheter placement before tumor sectioning is clearly visible in $\mathrm{C}$ and $\mathrm{D}$. These markers were used to assist fluorescence and autoradiographic images. Bar in C = $500 \mu \mathrm{m}$.

contrast, ${ }^{64} \mathrm{Cu}$-ATSM showed a pattern of distribution entirely different from the fluorinated nitroimidazoles. Generally, more intense ${ }^{64} \mathrm{Cu}$-ATSM uptake was observed in the peripheral rim of the tumor, with decreasing uptake toward the central tumor regions. ${ }^{64} \mathrm{Cu}$-ATSM uptake appeared to colocalize with areas of higher Hoechst 33342 intensity and less so with the areas of highest CA9 and pimonidazole staining intensity (Fig. 4).

\section{Rebinned Scatterplot Analysis}

Pixel values from coregistered autoradiographs and fluorescence images were rebinned and scatterplots generated as previously described (13). Representative scatterplots of Hoechst 33342, pimonidazole, and CA9 fluorescence intensity versus radiotracer pixel count are shown in Figure 5 and Supplemental Table 2. For ${ }^{18} \mathrm{~F}$-FMISO, ${ }^{18} \mathrm{~F}$-HX4, and ${ }^{18} \mathrm{~F}$-FAZA, there is a clear monotonically increasing relationship between pimonidazoleCA9 fluorescence and radiotracer uptake, together with a more complex but generally inverse relationship between Hoechst 33342 fluorescence and radiotracer uptake. However, the relationship between ${ }^{64} \mathrm{Cu}$-ATSM uptake and the fluorescence markers was different from those for the fluorinated nitroimidazoles. For ${ }^{64} \mathrm{Cu}$-ATSM, the highest uptake values were associated with the lowest values of pimonidazole-CA9 fluorescence and higher, rather than lower, values of Hoechst 33342 fluorescence (Fig. 5D).

To exclude the possibility that the observed distribution of ${ }^{64} \mathrm{Cu}$-ATSM was model-dependent, we performed the same experiment using 2 alternative xenograft models, HT29 and HCT116. Similarly to the SQ20b xenografts, higher ${ }^{64} \mathrm{Cu}$-ATSM uptake was observed in the peripheral tumor regions with decreasing uptake toward the tumor center. Figure 6 shows comparative coregistered fluorescence overlays and ${ }^{64} \mathrm{Cu}-\mathrm{ATSM}$ autoradiographs for all 3 xenograft models. The rebinned scatterplots show broadly similar relationships between the fluorescence markers and ${ }^{64} \mathrm{Cu}$-ATSM uptake - that is, lower CA9-pimonidazole and higher Hoechst 33342 fluorescence is associated with higher ${ }^{64} \mathrm{Cu}$-ATSM uptake.

\section{DISCUSSION}

This study directly compared the imaging characteristics and hypoxia selectivity of 4 hypoxia PET radiotracers under standardized experimental conditions. The principal tumor model used in this study (SQ20b) was selected because of its heterogeneous microenvironment and high hypoxia-inducible CA9 expression (Fig. 2).

This study was not designed to take into account the differing pharmacokinetics of each tracer. We used an 80- to 90-min postadministration imaging window for each tracer as an intermediate time between those commonly reported for the fluorinated nitroimidazoles $(90-180 \mathrm{~min})$ and ${ }^{64} \mathrm{Cu}-\mathrm{ATSM}(30$ $60 \mathrm{~min})(6,8,15-18)$. This single time point was selected to permit a direct comparison of the properties of each tracer without a major deviation from the currently used clinical imaging protocols for each. In the case of the fluorinated nitroimidazoles, increasing tumor-to-blood ratio over time is counteracted by deteriorating image quality due to reducing counting rate, which effectively limits the potential imaging window to between 90 and $180 \mathrm{~min}$ (19). However, uptake at this time period is assumed to reflect underlying $\mathrm{pO}_{2}$ with minimal confounding effects of residual tracer in the blood pool (20). The most appropriate imaging time for ${ }^{64} \mathrm{Cu}$-ATSM is less well established, with several studies indicating a wide range of potential values. Initial preclinical and early clinical studies evaluated tumor uptake of ${ }^{60} \mathrm{Cu}-$ and ${ }^{64} \mathrm{Cu}-$ ATSM between 30 and $60 \mathrm{~min}$ after administration $(15,16,21)$. Our own recent study using the human FaDu head and neck xenograft model found that, whereas absolute tumor uptake and tumor-to-muscle ratio continued to increase up to $18 \mathrm{~h}$ after administration of ${ }^{64} \mathrm{Cu}$-ATSM, no changes in the tumor distribution was observed over this period (22). Together, these data imply that

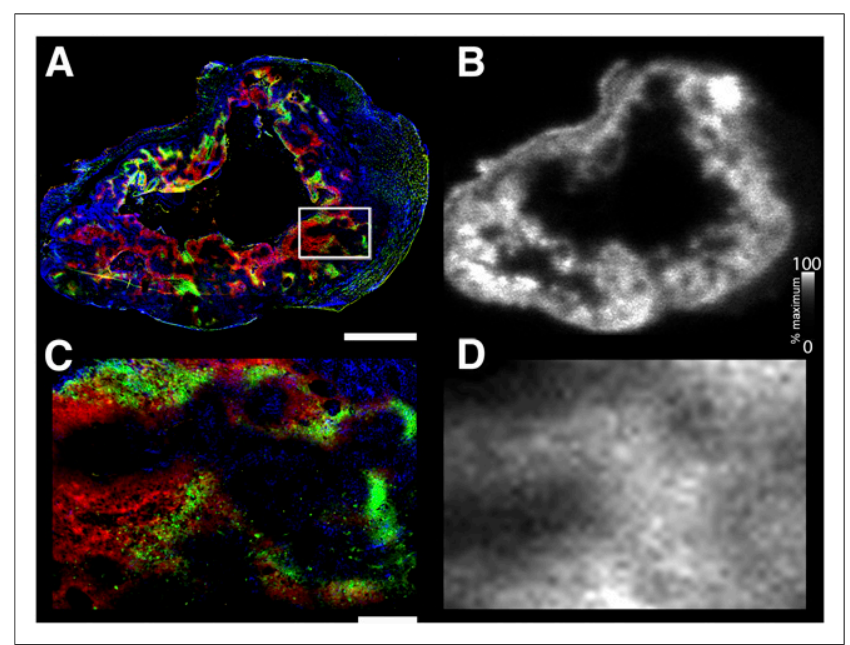

FIGURE 4. Distribution of ${ }^{64} \mathrm{Cu}-\mathrm{ATSM}$ versus immunofluorescence makers. Composite registered 3-color image showing Hoechst 33342 (blue), pimonidazole (green), and CA9 (red) is shown in A. Bar in A = 2 $\mathrm{mm}$. (B) Corresponding autoradiographic image of ${ }^{18} \mathrm{~F}$-FMISO distribution from same tumor section. Indicated region in $A$ is shown at high magnification in $C$ and $D$. Bar in $B=500 \mu \mathrm{m}$. 


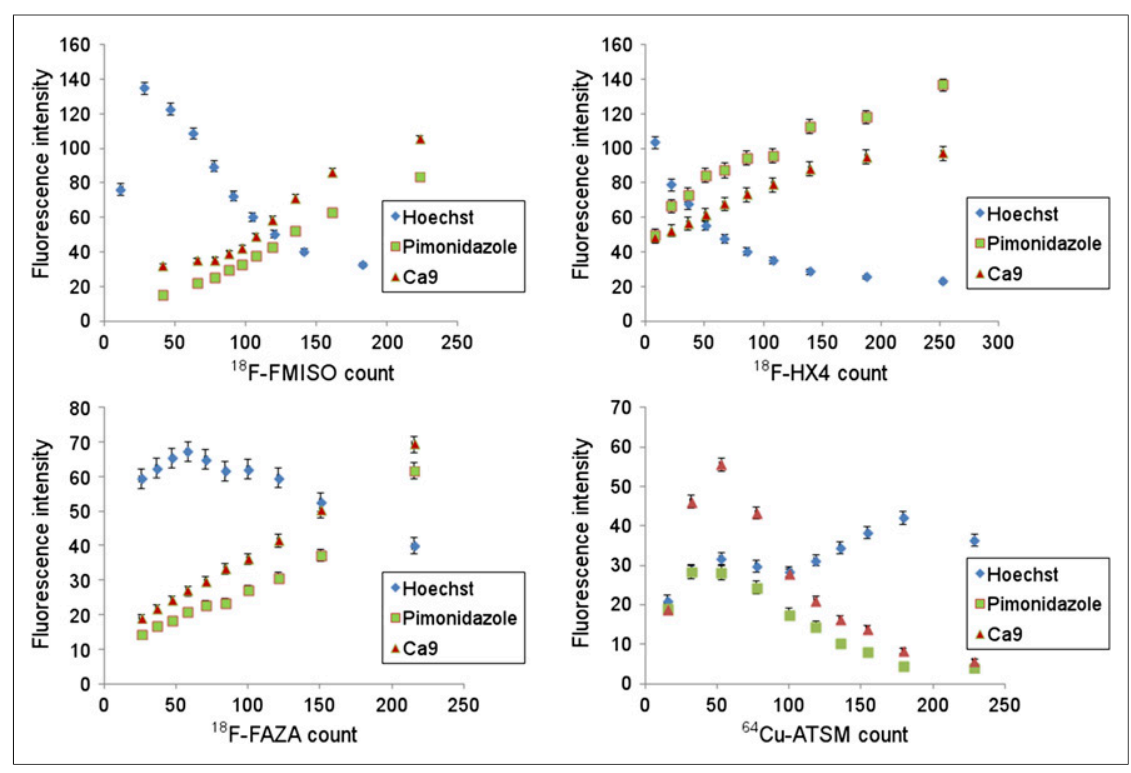

FIGURE 5. Rebinned scatterplots showing relationship between fluorescence markers Hoechst 33342 (blue), pimonidazole (green), CA9 (red) on ordinate, and indicated hypoxia radiotracer on abscissa. Mean and $95 \%$ confidence intervals of mean are shown.

the tumor distribution of ${ }^{64} \mathrm{Cu}-\mathrm{ATSM}$ at $80-90 \mathrm{~min}$ after injection is most likely a reasonable reflection of the hypoxia selectivity.

The imaging data presented here are in keeping with previous reports using single- or dual-tracer studies (Fig. 1; Table 1). ${ }^{18} \mathrm{~F}-$ FMISO and ${ }^{18} \mathrm{~F}-\mathrm{HX} 4$ showed broadly similar tumor uptake, with relatively lower abdominal ${ }^{18} \mathrm{~F}-\mathrm{HX} 4$ retention. ${ }^{18} \mathrm{~F}-\mathrm{FAZA}$ showed reduced absolute tumor and normal tissue uptake, compared with

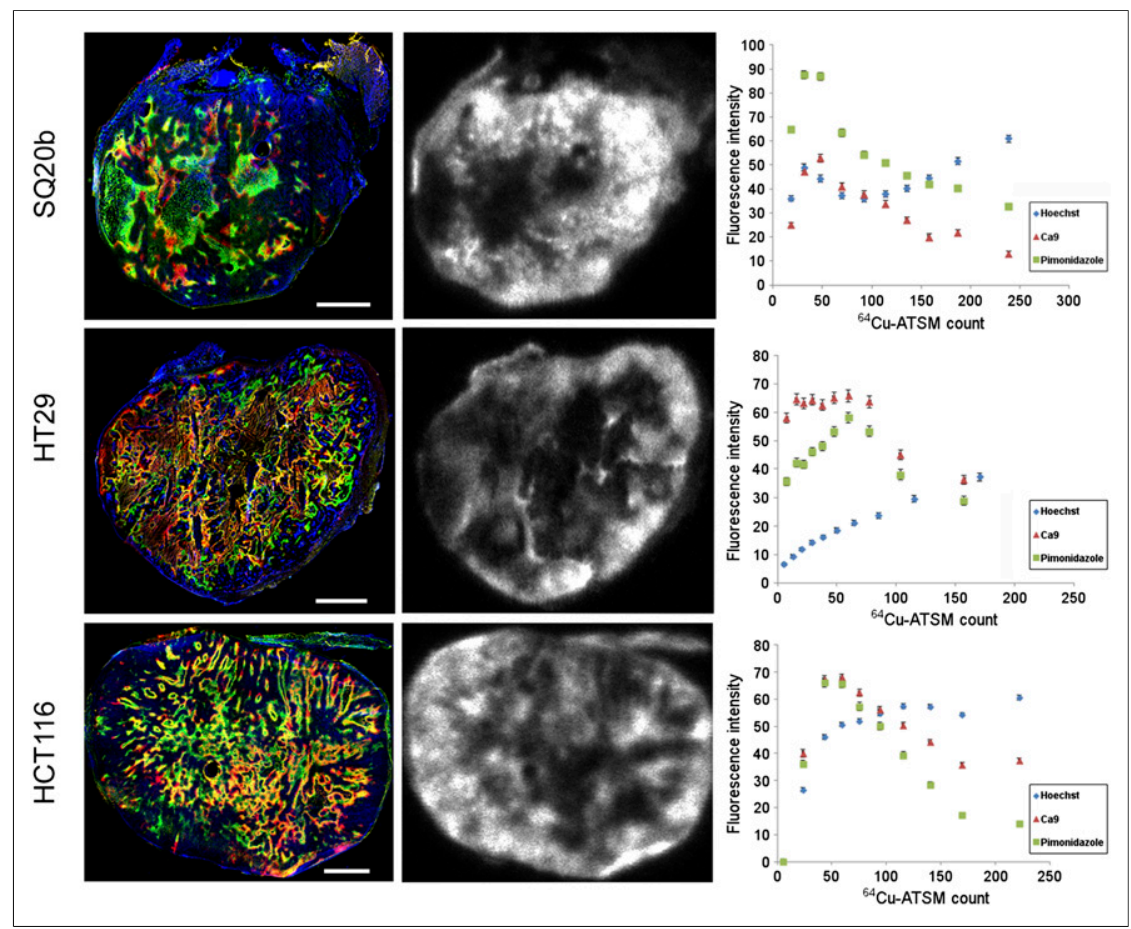

FIGURE 6. Distribution of ${ }^{64} \mathrm{Cu}-\mathrm{ATSM}$ versus immunofluorescence makers in 3 tumor models. Registered 3-color immunofluorescence images, corresponding autoradioraphy, and rebinned scatterplots from representative sections obtained from SQ20b (top), HT29 (middle), and HCT116 (bottom) xenograft tumors. Bar $=2 \mathrm{~mm}$.
${ }^{18} \mathrm{~F}-\mathrm{FMSIO}$ and ${ }^{18} \mathrm{~F}-\mathrm{HX} 4$, while retaining tumor image contrast. Tumor-to-muscle ratios were similar for each of the fluorinated nitroimidazoles, and no statistical difference was observed between either tracer (Supplemental Table 1). ${ }^{64} \mathrm{Cu}$-ATSM showed significantly greater tumor uptake and tumorto-muscle ratio than the fluorinated tracers, along with marked liver uptake and an absence of renal clearance. Autoradiographic determination of radiotracer distribution was compared with the histologic hypoxia markers pimonidazole and CA9 and also with the vascular perfusion marker Hoechst 33342. Although the distribution of the fluorinated nitroimidazoles all showed a monotonic positive correlation with both CA9 and pimonidazole, ${ }^{64} \mathrm{Cu}$-ATSM displayed a much more complex relationship with these markers (Figs. 5 and 6). In each of the 3 models examined, the lowest rebinned ${ }^{64} \mathrm{Cu}$-ATSM pixel values correlated with those with highest pimonidazole and CA9 expression. The inverse of this relationship was seen with Hoechst 33342, suggesting a relationship between uptake and vascular delivery of the tracer. However, the correlation between ${ }^{64} \mathrm{Cu}$-ATSM distribution and all of the histologic markers was clearly nonlinear.

Although pimonidazole and CA9 are commonly suggested as gold standards of tumor hypoxia, it is important to place these markers within the context of this study. The expression of CA9, although tightly regulated by the hypoxiainducible transcription factor $\mathrm{HIF} 1 \alpha$, cannot be assumed to be an exclusive marker of tumor hypoxia. Confounding factors include tissue-dependent and nonhypoxic regulation of expression, antigen shedding, and inability to reflect acute changes in $\mathrm{pO}_{2}(5,23,24)$. Pimonidazole is a 2-nitroimidazole compound, which has been widely validated as an immunohistochemical hypoxia marker, with reports of nonhypoxic uptake limited to central nervous system tissues only (25). It is structurally similar to the fluorinated tracers used in this study but unrelated to the (bis)thoisemicarbazone ATSM. The absolute uptake of pimonidazole in tumor hypoxic regions, and its correlation with other histologic hypoxia markers, may increase with time between administration and sacrifice, complicating the quantitative assessment of pimonidazole distribution (26). However, our use of both markers, coupled with the observation of a close correspondence between the 2, provides strong evidence of an underlying microregional hypoxic microenvironment and that both markers together can serve as a reasonable basis for comparison of PET tracer hypoxia specificity in the SQ20b model system (Fig. 2). 
The absence of a positive correlation between ${ }^{64} \mathrm{Cu}$-ATSM distribution, pimonidazole uptake, and CA9 expression requires some explanation. It is likely that the $\mathrm{pO}_{2}$ sensitivity and distribution of pimonidazole would correspond more closely to the other 2-nitroimidazoles than ${ }^{64} \mathrm{Cu}$-ATSM, which has previously been shown to have a complex relationship to pimonidazole distribution in other tumor models $(22,27,28)$. In addition, it has been suggested that ${ }^{64} \mathrm{Cu}$-ATSM uptake discriminates a wider range of hypoxic values than pimonidazole, leading to uptake in tumor regions that are not permissive for ${ }^{18} \mathrm{~F}-\mathrm{FMISO}$ (29). However, CA9 expression and pimonidazole bioreduction occur at different $\mathrm{pO}_{2}$ values $(<20$ and $<10 \mathrm{mmHg}$, respectively) $(30-32)$, and the low uptake of ATSM at either of these $\mathrm{pO}_{2}$ values casts doubt on the ability to interpret PET images of ATSM distribution as reflecting locoregional $\mathrm{pO}_{2}$ gradients. Recent studies by Bowen et al. have indicated that ${ }^{64} \mathrm{Cu}$-ATSM distribution may be governed principally by low tumor extracellular $\mathrm{pH}$ (pHe), rather than low pO2 exclusively (29), which may explain the prognostic significance of high ATSM uptake in certain tumor types (33). Although low tumor pO2 and low pHe may be expected to be found together in the same tumors, this relationship is not necessarily linear. It is possible that the reduction of tumor pHe via hypoxia-inducible enzymes (such as CA9, MCT4, or LDHA) could result in high pHe-mediated ATSM uptake in hypoxic tumors, but without matching localization with hypoxic microregions, as seen in the data presented in this study. For these reasons, ${ }^{64} \mathrm{Cu}$-ATSM may not be the optimal tracer in circumstances in which accurate delineation and mapping of the hypoxic tumor regions is of primary importance, such as image-guided intensity-modulated radiotherapy (34). However, the high tumor uptake of ${ }^{64} \mathrm{Cu}$-ATSM results in excellent image contrast, and accurate identification of the conditions permissive for ${ }^{64} \mathrm{Cu}$-ATSM retention in vivo could provide important information additional to that provided by other PET tracers.

\section{CONCLUSION}

${ }^{18} \mathrm{~F}-\mathrm{FMISO}$ and ${ }^{18} \mathrm{~F}-\mathrm{HX} 4$ demonstrated similar tumor uptake, with a slightly higher renal clearance distribution observed for ${ }^{18} \mathrm{~F}-\mathrm{HX} 4 .{ }^{18} \mathrm{~F}-\mathrm{FAZA}$ demonstrated the lowest tumor uptake and lowest background. ${ }^{64} \mathrm{Cu}$-ATSM demonstrated the highest tumor uptake and image contrast of the hypoxia tracers studied. Autoradiography and correlative histology confirm targeting of hypoxic tumor regions for the fluorinated 2-nitroimidazoles but cast doubt on the hypoxia-selectivity of ${ }^{64} \mathrm{Cu}$-ATSM.

\section{DISCLOSURE}

The costs of publication of this article were defrayed in part by the payment of page charges. Therefore, and solely to indicate this fact, this article is hereby marked "advertisement" in accordance with 18 USC section 1734. Technical services provided by the MSKCC Small Animal Imaging Core Facility, supported in part by NIH Small-Animal Imaging Research Program (SAIRP) grant R24 CA83084 and NIH Center grant P30 CA08748, are gratefully acknowledged. The latter grant also partially supports the MSKCC Research Animal Resource Center. This study was supported by the MSKCC-AstraZeneca partnership, and National Institutes of Health grants R01 CA138468. Dr. Carlin is funded in part by the Geoffrey Beene Cancer Research Foundation. No other potential conflict of interest relevant to this article was reported.

\section{ACKNOWLEDGMENTS}

We thank the Memorial Sloan-Kettering Cancer Center (MSKCC) Cyclotron-Radiochemistry Core Facility for assistance with isotope production and radiochemical synthesis. Assistance from the MSKCC Nuclear Pharmacy and MSKCC Molecular Cytology Core Facility is gratefully acknowledged.

\section{REFERENCES}

1. Thomlinson RH, Gray LH. The histological structure of some human lung cancers and the possible implications for radiotherapy. Br J Cancer. 1955;9:539-549.

2. Semenza GL. Hypoxia-inducible factors: mediators of cancer progression and targets for cancer therapy. Trends Pharmacol Sci. 2012;33:207-214.

3. Russell J, Carlin S, Burke SA, Wen B, Yang KM, Ling CC. Immunohistochemical detection of changes in tumor hypoxia. Int $J$ Radiat Oncol Biol Phys. 2009;73:1177-1186.

4. Le QT, Kong C, Lavori PW, et al. Expression and prognostic significance of a panel of tissue hypoxia markers in head-and-neck squamous cell carcinomas. Int J Radiat Oncol Biol Phys. 2007;69:167-175.

5. Swietach P, Hulikova A, Vaughan-Jones RD, Harris AL. New insights into the physiological role of carbonic anhydrase IX in tumour $\mathrm{pH}$ regulation. Oncogene. 2010;29:6509-6521.

6. Krohn KA, Link JM, Mason RP. Molecular imaging of hypoxia. J Nucl Med. 2008;49(suppl 2):129S-148S

7. Janssen HL, Hoebers FJ, Sprong D, et al. Differentiation-associated staining with anti-pimonidazole antibodies in head and neck tumors. Radiother Oncol. 2004; 70:91-97.

8. Carlin S, Humm JL. PET of hypoxia: current and future perspectives. $J$ Nucl Med. 2012;53:1171-1174.

9. Fujibayashi Y, Taniuchi H, Yonekura Y, Ohtani H, Konishi J, Yokoyama A. Copper-62-ATSM: a new hypoxia imaging agent with high membrane permeability and low redox potential. J Nucl Med. 1997;38:1155-1160.

10. Grierson JR, Link JM, Mathis CA, Rasey JS, Krohn KA. A radiosynthesis of fluorine-18 fluoromisonidazole. J Nucl Med. 1989;30:343-350.

11. Maier FC, Kneilling M, Reischl G, et al. Significant impact of different oxygen breathing conditions on noninvasive in vivo tumor-hypoxia imaging using $\left[{ }^{18} \mathrm{~F}\right]-$ fluoro-azomycinarabino-furanoside ( $\left.\left[{ }^{18} \mathrm{~F}\right] \mathrm{FAZA}\right)$. Radiat Oncol. 2011;6:165.

12. Oehler C, O'Donoghue JA, Russell J, et al. ${ }^{18} \mathrm{~F}$-fluromisonidazole PET imaging as a biomarker for the response to 5,6-dimethylxanthenone-4-acetic acid in colorectal xenograft tumors. J Nucl Med. 2011;52:437-444.

13. Carlin S, Pugachev A, Sun X, et al. In vivo characterization of a reporter gene system for imaging hypoxia-induced gene expression. Nucl Med Biol. 2009;36: 821-831.

14. Carlin S, Khan N, Ku T, Longo VA, Larson SM, Smith-Jones PM. Molecular targeting of carbonic anhydrase IX in mice with hypoxic HT29 colorectal tumor xenografts. PLoS ONE. 2010;5:e10857.

15. Lewis JS, Laforest R, Dehdashti F, Grigsby PW, Welch MJ, Siegel BA. An imaging comparison of ${ }^{64} \mathrm{Cu}$-ATSM and ${ }^{60} \mathrm{Cu}$-ATSM in cancer of the uterine cervix. J Nucl Med. 2008;49:1177-1182.

16. Dehdashti F, Grigsby PW, Lewis JS, Laforest R, Siegel BA, Welch MJ. Assessing tumor hypoxia in cervical cancer by PET with ${ }^{60} \mathrm{Cu}$-labeled diacetyl-bis(N4methylthiosemicarbazone). J Nucl Med. 2008;49:201-205.

17. Chen L, Zhang Z, Kolb HC, Walsh JC, Zhang J, Guan Y. ${ }^{18}$ F-HX4 hypoxia imaging with PET/CT in head and neck cancer: a comparison with ${ }^{18} \mathrm{~F}-\mathrm{FMISO}$. Nucl Med Commun. 2012;33:1096-1102.

18. Mortensen LS, Johansen J, Kallehauge J, et al. FAZA PET/CT hypoxia imaging in patients with squamous cell carcinoma of the head and neck treated with radiotherapy: results from the DAHANCA 24 trial. Radiother Oncol. 2012; 105:14-20.

19. Rasey JS, Koh WJ, Evans ML, et al. Quantifying regional hypoxia in human tumors with positron emission tomography of $\left[{ }^{18} \mathrm{~F}\right]$ fluoromisonidazole: a pretherapy study of 37 patients. Int J Radiat Oncol Biol Phys. 1996;36:417-428.

20. Hendrickson K, Phillips M, Smith W, Peterson L, Krohn K, Rajendran J. Hypoxia imaging with [F-18] FMISO-PET in head and neck cancer: potential for guiding intensity modulated radiation therapy in overcoming hypoxia-induced treatment resistance. Radiother Oncol. 2011;101:369-375.

21. Lewis JS, McCarthy DW, McCarthy TJ, Fujibayashi Y, Welch MJ. Evaluation of ${ }^{64} \mathrm{Cu}-\mathrm{ATSM}$ in vitro and in vivo in a hypoxic tumor model. $\mathrm{J}$ Nucl Med. 1999;40:177-183.

22. McCall KC, Humm JL, Bartlett R, Reese M, Carlin S. Copper-64-diacetyl-bis(N (4)-methylthiosemicarbazone) pharmacokinetics in FaDu xenograft tumors and 
correlation with microscopic markers of hypoxia. Int J Radiat Oncol Biol Phys. 2012;84:e393-e399.

23. Kaluz S, Kaluzová M, Liao SY, Lerman M, Stanbridge EJ. Transcriptional control of the tumor- and hypoxia-marker carbonic anhydrase 9: a one transcription factor (HIF-1) show? Biochim Biophys Acta. 2009;1795:162-172.

24. Shin KH, Diaz-Gonzalez JA, Russell J, Chen Q, Burgman P, Li XF, Ling CC. Detecting changes in tumor hypoxia with carbonic anhydrase IX and pimonidazole. Cancer Biol Ther. 2007;6:70-75.

25. Taghian A, Lespinasse F, Guichard ME. Radiosensitization by the combination of etanidazole (SR-2508) and pimonidazole (Ro 03-8799) in human tumor xenografts. Int J Radiat Oncol Biol Phys. 1991;21:1535-1540.

26. Isoda T, Engles J, Wahl R. Time dependence of hypoxia-specific accumulation of pimonidazole in rodent breast cancers [abstract]. J Nucl Med. 2010;51(suppl 2):228.

27. O'Donoghue JA, Zanzonico P, Pugachev A, et al. Assessment of regional tumor hypoxia using ${ }^{18} \mathrm{~F}$-fluoromisonidazole and ${ }^{64} \mathrm{Cu}$ (II)-diacetyl-bis(N4-methylthiosemicarbazone) positron emission tomography: Comparative study featuring microPET imaging, Po2 probe measurement, autoradiography, and fluorescent microscopy in the R3327-AT and FaDu rat tumor models. Int J Radiat Oncol Biol Phys. 2005;61:1493-1502.

28. Hansen AE, Kristensen AT, Jørgensen JT, et al. ${ }^{64} \mathrm{Cu}-\mathrm{ATSM}$ and ${ }^{18}$ FDG PET uptake and ${ }^{64} \mathrm{Cu}$-ATSM autoradiography in spontaneous canine tumors: compar- ison with pimonidazole hypoxia immunohistochemistry. Radiat Oncol. 2012; 7:89.

29. Bowen SR, van der Kogel AJ, Nordsmark M, Bentzen SM, Jeraj R. Characterization of positron emission tomography hypoxia tracer uptake and tissue oxygenation via electrochemical modeling. Nucl Med Biol. 2011;38:771780 .

30. Hammond EM, Giaccia AJ. The role of p53 in hypoxia-induced apoptosis. Biochem Biophys Res Commun. 2005;331:718-725.

31. Nordsmark M, Eriksen JG, Gebski V, Alsner J, Horsman MR, Overgaard J. Differential risk assessments from five hypoxia specific assays: the basis for biologically adapted individualized radiotherapy in advanced head and neck cancer patients. Radiother Oncol. 2007;83:389-397.

32. Kleiter MM, Thrall DE, Malarkey DE, Ji X, Lee DY, Chou SC, Raleigh JA. A comparison of oral and intravenous pimonidazole in canine tumors using intravenous CCI-103F as a control hypoxia marker. Int J Radiat Oncol Biol Phys. 2006;64:592-602.

33. Zhang $\mathrm{X}$, Lin $\mathrm{Y}$, Gillies RJ. Tumor $\mathrm{pH}$ and its measurement. J Nucl Med. 2010;51:1167-1170.

34. Ling CC, Humm J, Larson S, Amols H, Fuks Z, Leibel S, Koutcher JA. Towards multidimensional radiotherapy (MD-CRT): biological imaging and biological conformality. Int J Radiat Oncol Biol Phys. 2000;47:551-560.

\section{Errata}

In the article "Assessment of Tumoricidal Efficacy and Response to Treatment with ${ }^{18}$ F-FDG PET/CT After Intraarterial Infusion with the Antiglycolytic Agent 3-Bromopyruvate in the VX2 Model of Liver Tumor," by Liapi et al. ( $\mathrm{J} \mathrm{Nucl} \mathrm{Med.} \mathrm{2011;52:225-230),} \mathrm{there} \mathrm{was} \mathrm{a} \mathrm{typographic} \mathrm{error} \mathrm{in} \mathrm{one} \mathrm{of} \mathrm{the} \mathrm{grants} \mathrm{mentioned} \mathrm{in} \mathrm{the} \mathrm{"Acknowledg-}$ ments" section. The study was supported by NIH/NCI grant RO1 CA100883-01, not CA100882-01. The authors regret the error.

In the article "Radioimmunotherapy with ${ }^{177}$ Lu-DOTA-Rituximab: Final Results of a Phase I/II Study in 31 Patients with Relapsing Follicular, Mantle Cell, and Other Indolent B-Cell Lymphomas," by Forrer et al. ( $\mathrm{J}$ Nucl Med. 2013;54:1045-1052), there was an error in the reported mean doses to the 20 patients. The correct whole-body dose is $0.32 \mathrm{mGy} / \mathrm{MBq}$, and the correct absorbed dose to the red marrow is $0.36 \mathrm{mGy} / \mathrm{MBq}$. The authors regret the error.

In Table 1 of "The SNMMI and EANM Practice Guideline for Small-Bowel and Colon Transit 1.0," by Maurer et al. ( J Nucl Med. 2013;54:2004-2013), administered activity was incorrectly given in units of MBq/kg (mCi/kg) instead of $\mathrm{MBq}(\mathrm{mCi})$. The authors regret the error. 